\title{
Movement and focused attention: A failure to replicate
}

\author{
ARTHUR F. KRAMER and MING-PO THAM \\ Beckman Institute for Advanced Science and Technology, University of Illinois, Champaign, Illinois \\ and \\ YEI-YU YEH \\ University of Wisconsin, Madison, Wisconsin
}

\begin{abstract}
Our original goal was to explore the nature of the grouping-by-movement phenomenon reported by Driver and Baylis (1989). In their studies, distractors that moved in common with a centrally located target had a larger influence on focused-attention performance than did more proximate but stationary distractors. These results seemed particularly important since they suggested, contrary to the predictions of space-based models of attention, that attention could be allocated to noncontiguous regions of the visual field. Their results also suggested mandatory processing of stimuli with common motion. Unfortunately, we were unable to replicate this grouping-bymovement effect. In the conditions of Experiment 1 in which we replicated Driver and Baylis's methodology, stationary distractors produced a larger response-compatibility effect than did the more distant distractors that moved in common with the target. In Experiment 2, we redundantly coded the centrally located target and the far distractors with common movement and color. However, the results were identical to those obtained in Experiment 1. The stationary near distractors that appeared in a different color from the target and the far distractors produced the largest response-compatibility effect. In a final experiment, we attempted to compensate for the reduced acuity of the moving distractors by adjusting their size by a cortical magnification factor. However, even with this manipulation, we found a larger response-compatibility effect for the stationary near distractors than for the moving distant distractors. Our results suggest that subjects are capable of selectively processing a target item that moves in common with distractors.
\end{abstract}

In the present experiments, we investigated the influence of three different grouping cues-movement, proximity, and color-on the distribution of attention to a limited area of the visual field. More specifically, we were interested in the degree to which color and movement could be used to offset the influence of proximity on focused attention.

Our interest in the effects of different cues on attentional control stems from the apparent incompatibilities between two classes of attentional models. Space-based models suggest that, at any one time, attention is allocated to a limited but contiguous region of visual space. Stimuli within this region receive full processing, while stimuli that reside outside of the focus of attention are excluded from processing. There are a number of variations to this class of model, such as spotlights, zoom lenses, and gradients (Broadbent, 1982; Downing, 1988; C. W. Eriksen \& St. James, 1986; C. W. Eriksen \& Yeh, 1985; Jonides, 1983; Mangun \& Hillyard, 1987; Posner,

The research was supported by Office of Naval Research Grant N-00014-89-J-1493, monitored by Harold Hawkins. The authors wish to thank Charles Eriksen and Steven Yantis for their helpful comments on an eariler draft of this article. Requests for additional information or reprints should be addressed to Arthur F. Kramer, Department of Psychology, University of Illinois, 603 East Daniel St., Champaign, Il 61820 .
Snyder, \& Davidson, 1980; Remington \& Pierce, 1984; Shulman, Remington, \& McLean, 1979; Tsal \& Lavie, 1988). Although there are several differences among these models, they all suggest that space provides the medium within which visual attention operates and that selective attention is most effective when location cues are available. Space-based models have been supported by results obtained in divided-attention, response-compatibility, and spatial-priming paradigms (Bashinski \& Bacharach, 1980; B. A. Eriksen \& C. W. Eriksen, 1974; C. W. Eriksen \& Hoffman, 1972, 1973; Gatti \& Egeth, 1978; Hagenaar \& van der Heijden, 1986; Hoffman, Nelson, \& Houck, 1983; Posner, 1980).

Object-based models differ from space-based models in their emphasis on the relative importance of different grouping cues. Thus, while space-based models suggest that proximity is the primary cue for the grouping of stimuli in the visual field, object-based models argue that proximity does not have any special status for grouping but instead is one of a number of cues that can be used to parse the visual field. For example, Neisser (1967) proposed a two-stage model of attention in which the visual field is initially and preattentively segmented into separate figural units or objects on the basis of Gestalt properties such as continuity, proximity, color, and movement. In the second stage, focal attention is then used to analyze 
specific figural units in more detail (for more recent versions of object-based models, see Duncan, 1984; Duncan \& Humphreys, 1989; Kahneman \& Henik, 1977, 1981; Kahneman \& Treisman, 1984; Kramer \& Jacobson, 1991; Prinzmetal, 1981).

Evidence in support of object- or grouping-based models has been provided by studies that have endeavored to hold the proximity between stimuli constant while varying other cues that may promote grouping. In one such study, Duncan (1984) instructed subjects to identify two attributes from a single object or one attribute on each of two separate objects. Spatial proximity between the same- and different-object conditions was controlled by superimposing the two objects. The results indicated that, in general, the identification of two attributes on a single object was more accurate than the identification of one attribute on each of two separate objects (see also Lappin, 1967; Treisman, Kahneman, \& Burkell, 1983; Yntema, 1963). Other studies have also shown that attentional processes can be influenced by such cues as contour, color, form, orientation, and spatial frequency (Banks, Bodinger, \& Ilige, 1974; Banks \& Prinzmetal, 1976; Barber \& Folkard, 1972; Beck \& Ambler, 1973; Bundersen \& Pedersen, 1983; Davis, Kramer, \& Graham, 1983; Humphreys, 1981; LaBerge \& Brownstein, 1974; Pomerantz \& Pristach, 1989; Prinzmetal, 1981; von Wright, 1968).

It is important to note, however, that although it is now clear that cues other than proximity can be used to influence attentional selectivity, there is still abundant evidence to suggest that spatial cues have a special status in attentional control. Treisman and colleagues (Treisman \& Gelade, 1980; Treisman \& Gormican, 1988) have argued, on the basis of data from visual search tasks, that although individual features are registered preattentively without knowledge of their location, the integration of features into unified percepts is mediated by their location. Nissen (1985) found that when subjects were to report the shape and location of items cued by color, localization of cued items was required for correct selection of the corresponding shapes. Thus, it appears that localization of the items mediated the integration of the cued (color) and reported (shape) features. Tsal and Lavie (1988) had subjects search for a letter in a specified color or specified shape in a briefly presented array of nine letters. Reports of letters other than the targets occurred more frequently for letters close to the targets than for letters in the same color or the same shape as the target. They concluded from these results that "the direction of attention to a relevant spatial location seems to be a general and mandatory process that takes place irrespective of the dimension according to which the stimulus was initially selected for processing" (Tsal \& Lavie, 1988, p. 19). Finally, Garner (1987) found that location, but not color, cues enhanced performance in a series of contingent classification studies. The results of these studies appear to present a strong case for the uniqueness of location as a cue for selective and divided attention.
An interesting counterexample has been provided by Driver and Baylis (1989). In a series of studies, subjects were instructed to respond to a centrally located target and ignore two surrounding distractors on each side of the target. The distractors could either be compatible with the response of the target, incompatible with the response of the target, or neutral with respect to the response of the target. On a particular trial, one set of distractors (e.g., those located either near or far from the target in the fivestimulus array) was either response-compatible or responseincompatible with the target while the other set of distractors was neutral. Thus far, the task resembles the response-compatibility paradigm (B. A. Eriksen \& C. W. Eriksen, 1974). However, Driver and Baylis added a novel twist that enabled them to examine whether or not the effects of spatial location could actually be overridden by another grouping cue, movement. This was accomplished by moving the target and the far distractors in the same direction and with the same velocity while maintaining the position of the near distractors. It is important to note that even after the movement, the stationary near distractors were closer to the target than the far distractors.

Space-based models of attention, as well as the studies described above that support the notion of a special status for spatial location, predict that the near distractors should produce a larger response-compatibility effect than should the far distractors. A larger near-distractor effect would also be predicted on the basis of acuity differences between near and far distractors, since target/distractor proximity and acuity were confounded by the use of the horizontal array of letters. On the other hand, object- and grouping-based models would argue that common movement may serve to group the centrally located target with the far distractors, thereby producing a larger responsecompatibility effect for the far distractors. The results were consistent with predictions of object-based models. Reaction times (RTs) were longer and error rates were higher when the far distractors were incompatible with the response of the target.

At first glance, these results may not seem surprising since other studies have also found grouping by cues other than proximity (Duncan, 1984; Harms \& Bundersen, 1983; Humphreys, 1981; Kramer \& Jacobson, 1991; Kramer, Wickens, \& Donchin, 1985; Lappin, 1967; Treisman et al., 1983; Yntema, 1963). However, it is important to note that, in these other studies, the investigators held proximity constant while varying other grouping cues. In the Driver and Baylis (1989) studies, grouping by common movement actually overrode grouping by proximity, thereby casting doubt on Tsal and Lavie's (1988) claim that attention to a relevant spatial location is a general and mandatory process. Instead, the fact that subjects in Driver and Baylis's tasks had unlimited time to focus their attention on the target location prior to the beginning of each trial suggests that common movement, rather than location, may be mandatorily processed. 


\section{EXPERIMENT 1}

In an effort to examine the generality and strength of the grouping-by-movement phenomenon, we began our experiments by systematically varying the proximity between the centrally located target and the near distractors. In one version of the response-compatibility task, we replicated, with minor differences, Driver and Baylis's (1989) Experiment 1. In this version, the center-tocenter distance between the target and the near distractors was $0.6^{\circ}$ of visual angle, and the distance between the target and the far distractors was $1.2^{\circ}$. In the second version of the task, we moved the near distractors closer to the target while maintaining the distance between the target and the far distractors. In this case, the center-tocenter distance between the target and the near distractors was $0.40^{\circ}$ of visual angle. The two versions of the task are represented in Figure 1. On the basis of previous studies of grouping by proximity, this manipulation should serve to increase the grouping of the target with the near distractors (Fryklund, 1975; Pomerantz \& Schwaitzberg, 1975). The target and the far distractors moved in the same direction and with the same velocity in both versions of the response-compatibility task.

If grouping by common movement is mandatory, the manipulation of the distance between the target and the near distractors should have little effect on the size of the response-compatibility effect produced by the far distractors. Thus, the results in both versions of the task should be consistent with those obtained by Driver and Baylis (1989): far distractors should produce a larger response-
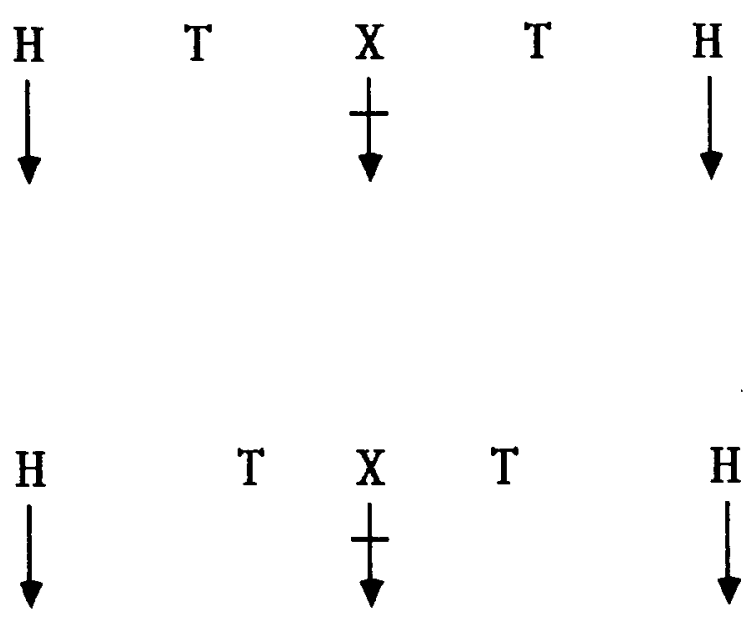

Figure 1. A graphic illustration of the baseline conditions in the experiments. In each of the experiments, the moving stimuli were equally likely to move up or down on any trial. The position of the fixation cross, which was present prior to the five letter stimuli, is also illustrated. The two letter strings represent, from top to bottom, the DB and proximate conditions in Experiment 1. The stimulus string presented in Experiment 2 was the same as that presented in the first row. However, in addition to the movement, the target and the far distractors were also presented in one color and the near distractors were presented in another color. compatibility effect than should near distractors. If, on the other hand, the strength of grouping depends on the relative salience of the proximity and movement cues, decreasing the distance between the target and the near distractors should increase the response-compatibility effect for the near distractors while simultaneously decreasing the response-compatibility effect for the far distractors.

\section{Method}

Subjects. Twelve right-handed students ( 7 males and 5 females) from the University of Illinois participated as subjects in the experiment. The subjects ranged in age from 18 to 23 years. All of the subjects had normal or corrected-to-normal vision. Each subject was paid $\$ 4.50$ per hour for participation in the experiment.

Stimuli and Apparatus. The stimuli used in Experiment 1 were the uppercase letters $H, T, X, Y, S$, and $C$. Each display was composed of a horizontal string of five letters. The target was the middle letter in this string. The letters $H$ and $T$ were used only as neutral flankers. Thus, these letters never served as targets. The letters $\mathrm{X}, \mathrm{Y}, \mathrm{S}$, and $\mathrm{C}$ were used as targets as well as response-compatible and response-incompatible flankers. The letters $X$ and $Y$ belonged to one response class, and the letters $S$ and $C$ belonged to the other response class. Thus, the subjects depressed one response button when $\mathrm{X}$ or $\mathrm{Y}$ were targets and another response button when $\mathrm{S}$ or $C$ were targets.

The subjects viewed the stimuli binocularly at a distance of $90 \mathrm{~cm}$ from the display. To ensure a fixed eye-to-screen distance, a chinrest was used by each subject. At that distance, each of the letters subtended a visual angle of $0.25^{\circ}$ horizontally and $0.38^{\circ}$ vertically. Movement of the stimuli was accomplished by presenting the letters at a different screen position in each of seven frames. Each frame remained on the monitor for $14.3 \mathrm{msec}$. Thus, the total presentation time of the stimuli was $100 \mathrm{msec}$. The vertical position of each frame was offset by $0.087^{\circ}$, for a total movement distance of $0.52^{\circ}$ in the seven frames. The target and the far flankers had an equal probability of moving up or down. Within each trial, movement was in one direction only. When moving up, the letters appeared initially $0.26^{\circ}$ below the fixation point; when moving down, the letters appeared $0.26^{\circ}$ above fixation. Whether moving up or down, the target letter appeared at fixation on the fourth frame. The fixation point was centered on the display and appeared for $500 \mathrm{msec}$ prior to the presentation of the letters. The five letters were horizontally aligned on the first frame of each trial.

The horizontal spacing of the five letters and the distance between the near flankers and the target differed across experimental conditions. In the Driver and Baylis (1989) replication condition, the center-to-center and minimum edge-to-edge horizontal separations between the five letters on the first frame was $0.60^{\circ}$ and $0.35^{\circ}$, respectively. Henceforth, this condition will be referred to as the $D B$ condition. Given that only the centrally located target and the far flankers moved during the seven frames, the final distance between the near flankers and the target increased to $0.80^{\circ}$. The distance between the target and the far flankers remained constant at $1.2^{\circ}$ since the far flankers moved with the target. Thus, even after the movement, the near flankers were closer to the target than were the far flankers. In the proximate condition, the center-to-center and minimum edge-to-edge separations between the target and the near flankers on the first frame were $0.40^{\circ}$ and $0.15^{\circ}$, respectively. The separation between the target and the far flankers was the same as in the DB condition ( $1.2^{\circ}$ center to center). Thus, the separation between the target and the near flankers was reduced and the separation between the near flankers and the far flankers was increased relative to the DB conditions. Given that only the centrally located target and the far flankers moved during the seven frames, the final distance between the near flankers and the target increased to $0.65^{\circ}$. The distance between the target and the far flankers re- 
mained constant at $1.2^{\circ}$. In both the DB and the proximate conditions, the near flankers were always repetitions of the same letter, as were the far flankers. One set of flankers, near or far, was either compatible or incompatible with the response of the target; the other set of letters, near or far, was neutral with respect to the response of the target.

The stimuli were generated on a Dell System 200 computer with 640K available RAM and displayed on a Dell VGA Color Plus monitor. Letters appeared in white (luminance $24.01 \mathrm{~cd} / \mathrm{m}^{2}$ ) against a black background (luminance $0.44 \mathrm{~cd} / \mathrm{m}^{2}$ ). The subjects depressed the $A$ and $L$ keys on the Dell keyboard to respond to the stimuli. Response key was counterbalanced across subjects. Response time was accurate to $1 \mathrm{msec}$.

Design. The experiment was a within-subject three-way factorial. The factors included compatibility (compatible and incompatible flankers), distance (near and far flankers), and stimulus arrangement (DB and proximate conditions). A separate baseline condition was also included for both the DB and the proximate versions of the task. In the baseline condition, all of the flankers were neutral. The first two factors, compatibility and distance, and the baseline condition were represented in each of the experimental blocks. The third factor, stimulus arrangement, served as a blocking factor. Six subjects performed in the first two sessions of the DB conditions and in the third and fourth sessions of the proximate conditions. The other 6 subjects performed in the first two sessions of the proximate conditions and in two sessions of the DB conditions.

Procedure. Experiment 1 was performed in four sessions over a period of 4 consecutive days. Each of the sessions lasted approximately $1 \mathrm{~h}$ and consisted of six blocks of 100 trials. On Days 1 and 3 , the first two blocks were treated as practice and were not analyzed with the rest of the data.

The subjects' task was to attend to the center of the display and press one button if the centrally located target was an X or a $\mathrm{Y}$ and another button if the target was an S or a $C$. Both speed and accuracy were emphasized. Trials were self-paced. The subjects depressed the space bar on the keyboard once they had fixated the

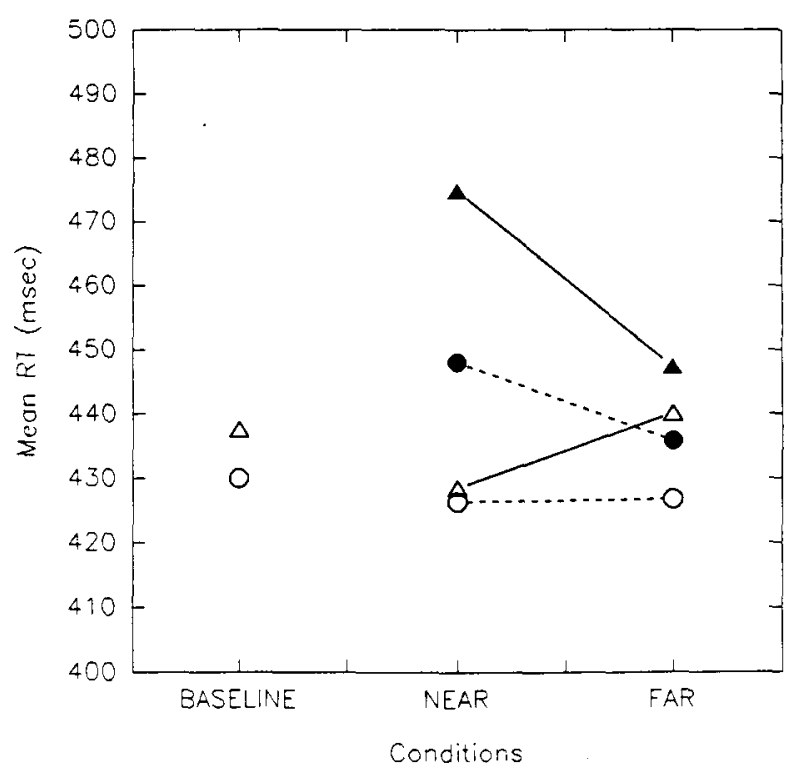

Figure 2. Average correct reaction times for each of the conditions in Experiment 1. Proximate conditions are coded by triangles; DB conditions are coded by circles. Filled symbols indicate incompatible conditions; unfilled symbols indicate compatible conditions. The DB baseline condition is coded by an unfilled circle; the proximate baseline condition is coded by an unfiled triangle.

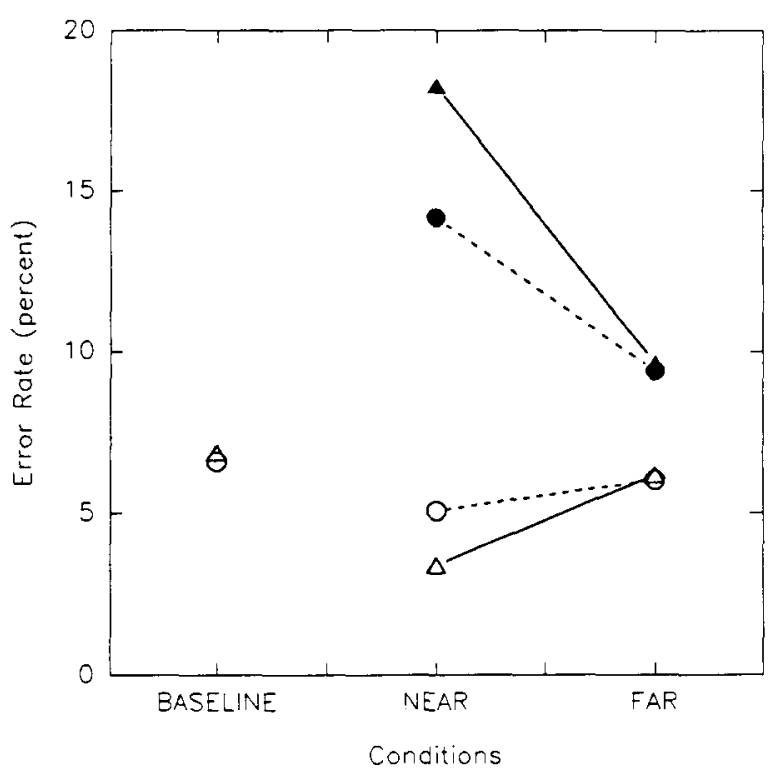

Figure 3. Average error rates for each of the conditions in Experiment 1. Proximate conditions are coded by triangles; DB conditions are coded by circles. Filled symbols indicate incompatible conditions; unflled symbols indicate compatible conditions. The DB baseline condition is coded by an unfilled circle; the proximate baseline condition is coded by an unfilled triangle.

"space-bar" prompt on the center of the display. A fixation cross was then presented in the center of the display for $500 \mathrm{msec}$. The five letters were then presented in seven successive frames of $14.3 \mathrm{msec}$, for a total duration of $100 \mathrm{msec}$. The trial was terminated with the subject's response. The subjects could start the next trial $2,500 \mathrm{msec}$ after the beginning of the previous trial.

Following each block of trials, the subjects were presented with a historical summary of their average reaction times and accuracy for each of the preceding blocks. This trend information was displayed graphically.

\section{Results}

The mean correct RTs and error rates are presented in Figures 2 and 3, respectively. The RTs and error rates were analyzed in two different ways. First, in an effort to provide a systematic comparison between the experimental conditions, we describe the results of three-way repeated measures analyses of variance (ANOVAs) performed on the mean RT and error data (compatibility $\times$ distance $\times$ stimulus arrangement). Second, we evaluated the relative costs and benefits of the experimental conditions relative to the neutral baselines separately for the DB and proximate versions of the response-compatibility task.

We begin by describing the results of the ANOVAs. Targets that were surrounded by response-incompatible distractors were responded to more slowly $[F(1,11)=$ $32.35, p<.01]$ and with a higher error rate $[F(1,11)=$ $45.79, p<.01]$ than were targets that were surrounded by response-compatible distractors. Second, RTs were faster $[F(1,11)=29.42, p<.01]$ and error rates were lower $[F(1,11)=11.7, p<.01]$ when the responsecompatible and response-incompatible distractors were located at the far positions. Third and more importantly we 
found a significant two-way interaction between compatibility and distance for both RT $[F(1,11)=57.19$, $p<.01]$ and error rate $[F(1,11)=19.84, p<.01]$. The difference between compatible and incompatible conditions was larger when the response-compatible and response-incompatible distractors were located next to the target than when they were located at the outer positions of the five-letter string.

Finally, of relevance to the comparison between the DB and the proximate versions of the task were the significant interactions between stimulus arrangement and compatibility [RT, $F(1,11)=8.15, p<.05$; error rate, $F(1,11)=8.29, p<.05]$ and a three-way interaction among stimulus arrangement, compatibility, and distance $[\mathrm{RT}, F(1,11)=25.51, p<.01$; error rate, $F(1,11)=$ 13.93, $p<.01]$. Post hoc comparisons indicated that the compatibility effect was larger at the near locations for the proximate version than for the DB version of the task. ${ }^{1}$ This effect is consistent with the relative-salience hypothesis: moving the inner flankers closer to the target (proximate condition) was predicted to increase the responsecompatibility effect relative to the DB version of the task in which the spacing between the target and inner distractors was the same as the spacing between the inner distractors and the outer distractors.

A series of Bonferoni $t$ tests were performed to compare the experimental conditions with the baselines in the DB and proximate conditions. All reported effects were significant at $p<.05$. In the DB version of the task, performance costs were significant for the near incompatible condition. RTs were slower and error rates were higher in the near incompatible condition than in the baseline condition. Error rates were also larger than the baseline for the far incompatible condition. However, the costs were larger for both RTs and error rates at the neardistractor distances than they were at the far-distractor distances. RTs and error rates did not differ from baseline in the compatible conditions.

In the proximate version, performance costs were obtained in the near incompatible condition. RTs and error rates were increased in the near incompatible condition relative to those in the baseline condition. Error rates were larger and RTs were longer for the far incompatible conditions than for the baseline condition. The costs were larger for both RTs and error rates at the near-distractor distances than they were at the far-distractor distances. Finally, performance was facilitated in the near compatible condition. Error rates were reduced in this condition relative to those in the baseline condition.

\section{Discussion}

The results clearly support the relative-salience hypothesis over the mandatory-processing hypothesis. ${ }^{2}$ Decreasing the distance between the near distractors and the target in the proximate condition, relative to that in the DB condition, increased the response-compatibility effect at the near location. Thus, it would appear that the processing of common movement is not mandatory, at least in situations in which subjects have ample time to focus their attention on the target location.

However, surprisingly, we were unable to replicate the response-compatibility effects that were obtained by Driver and Baylis (1989). In their experiments, common movement overrode the effects of proximity such that the far distractors that moved in the same direction and with the same velocity as the target produced larger responsecompatibility effects than did the near distractors. It is quite clear from an inspection of the mean RTs and error rates in Figures 2 and 3, as well as from the analyses presented above, that our results were quite disparate from those obtained by Driver and Baylis. In fact, in the DB condition, we found exactly the opposite pattern of results: the response-compatibility effect was larger at the neardistractor locations than at the far-distractor locations. Thus, in our study, common movement did not encourage grouping of the target with the far distractors.

What could be the reason for this failure to replicate the grouping-by-movement results obtained by Driver and Baylis? The differences in methodology between Driver and Baylis's Experiment 1 and our experiment were very minor. Driver and Baylis accomplished the movement of the target and the far distractors in five frames $(20 \mathrm{msec}$ per frame), whereas we moved the stimuli in seven frames (14.3 msec per frame). The total time for the movement and the distance moved was identical. Another difference was the presentation of error feedback. Driver and Baylis flashed the word wrong following an incorrect response. In our study, we graphically presented mean correct $R T$ and error feedback after each block of 100 trials. Neither of these methodological differences would be expected to produce the drastic differences in performance that were obtained in the two sets of studies.

A more plausible alternative for the failure to replicate the grouping-by-movement results obtained by Driver and Baylis might be the addition of the proximate version of the task in our experiment. Six of our subjects performed the proximate version of the task during Sessions 1 and 2 and the DB replication during Sessions 3 and 4. The other 6 subjects performed the DB version in Sessions 1 and 2 and the proximate version of the task during Sessions 3 and 4. It is possible that the 6 subjects who performed the proximate version first may have adopted a strategy in which the target and the near distractors were processed as a unitary group. If such a strategy was transferred to their performance in the DB version on Days 3 and 4 , it may have considerably weakened any groupingby-movement effects. However, this should not be the case for the other 6 subjects, who performed the DB version of the task during the first two sessions.

In an effort to examine this possibility, we performed a split-plot ANOVA in which the order of administration of the proximate and DB versions of the task served as a between-subjects factor. Neither the main effect of order or its interaction with the other factors attained statistical significance. Thus, it appears that the order of presentation of the two versions of the response-compatibility task 
did not influence the degree to which grouping by movement occurred.

At this point, we were quite perplexed as to the reason for our failure to replicate the Driver and Baylis (1989) effects. We decided that a reasonable strategy would be to try to enhance the grouping of the target with the far distractors by redundantly coding these stimuli with both common movement and color. Thus, although movement alone may be incapable of overriding the effects of proximity in focused attention, the redundant coding of the target and far distractors may serve the same purpose.

\section{EXPERIMENT 2}

Color has been used successfully in a number of focused-attention paradigms to segregate distractors from targets. For instance, Harms and Bundersen (1983) instructed subjects to respond to a centrally located target and ignore surrounding distractors. The distractors could either be compatible with the response of the target or incompatible with the target's response. Consistent with previous studies, response-incompatible distractors produced longer RTs and higher error rates than did responsecompatible distractors. However, the performance costs incurred with the incompatible distractors were reduced when the distractors appeared in a different color from the target. Humphreys (1981) found a similar grouping effect when subjects were required to respond to a target in a precued location that was flanked by a responseincompatible distractor. Subjects were able to ignore the distractor if it consistently appeared in a color different from the target even when the distractor was located within $0.5^{\circ}$ of the target (see also Kramer \& Jacobson, 1991). In summary, these studies suggest that color can provide an effective cue for selective attention such that the level of detection and identification performance improves beyond that obtained when only spatial cues are available.

It is important to note, however, that, in these studies, the spatial separation between stimuli was fixed while the efficacy of color cuing was examined. In Experiment 2, we examined whether or not stimuli that are redundantly coded by movement and color will be grouped together despite an alternative grouping suggested by proximity information. Thus, in essence, we are asking whether color and movement cues can be used to override grouping by proximity.

This was accomplished by presenting the target and the far flankers in one color and presenting the near flankers in another color. Thus, the target and the far flankers were redundantly coded with common movement and color. Furthermore, we capitalized on previous research (Humphrey, 1981) that has shown that consistent color coding provides more effective grouping than does varied coding. For any particular subject, one color was used to code the target and the far flankers and another color was used to code the near distractors throughout the experiment.

\section{Method}

Subjects. Twelve right-handed students (5 males and 7 females) from the University of Illinois participated as subjects in the experiment. The subjects ranged in age from 18 to 29 years. All of the subjects had normal or corrected-to-normal vision and obtained a perfect score on the Isharia Color Blindness Test (1989). Each subject was paid $\$ 4.50$ per hour for participation in the experiment.

Stimuli and Apparatus. The stimuli and apparatus were the same as those in Experiment 1, with the following exceptions. First, only the $\mathrm{DB}$ condition with response-compatible, response-incompatible, and neutral flankers was run. In this condition, the center-to-center distance between the target and the near flankers was $0.60^{\circ}$ of visual angle, as was the distance between the near flankers and the far flankers. Second, in Experiment 2, the target and the far flankers were redundantly coded by movement and color. Thus, in addition to moving in the same direction and with the same velocity as the target, the far flankers were also presented in the same color as the target. The static near flankers were presented in a different color. Red and green were used to code the letters. For half of the subjects, the target and the far flankers were presented in red and the near flankers were presented in green; for the other half of the subjects, the target and the far flankers were presented in green and the near flankers were presented in red. Thus, within subjects, color was consistently mapped to target and flanker positions. A subjective comparison of these colors with the Munsell color chips yielded the following Munsell values: red, hue $=5 R$, value $=6$, chroma $=10 ;$ green, hue $=10 \mathrm{GY}$, value $=6$, chroma $=10$. The background color was light gray.

Design. The experiment was a within-subject three-way factorial. The factors included compatibility (compatible and incompatible flankers), distance (near and far flankers), and color (red and green targets and far flankers). A baseline condition in which all of the flankers were presented in the same color and were neutral with respect to the response of the target was also included in the experiment.

Procedure. The experiment was performed in two sessions held on consecutive days. Each of the sessions lasted approximately $1 \mathrm{~h}$ and consisted of six blocks of 100 trials. The first two blocks on Day 1 were treated as practice and were not analyzed with the rest of the data. The experimental procedures were identical to those employed in Experiment 1.

\section{Results}

The mean correct RTs and error rates are presented in Figures 4 and 5, respectively. The RTs and error rates were analyzed in two different ways. First, in an effort to provide a systematic comparison among the experimental conditions, we submitted the data to two-way repeated measures ANOVAs (compatibility $x$ distance). ${ }^{3}$ Second, we evaluated the costs and benefits of the experimental conditions relative to the neutral baseline conditions.

Consistent with the figures, RTs were slower and error rates were higher when the target was surrounded by the response-incompatible distractors than when it was flanked by the compatible distractors $[\mathrm{RT},[F(1,11)=$ $15.6, p<.01$; error rate, $F(1,11)=14.98, p<.01]$. RTs were also slower when the response-compatible and response-incompatible distractors were located at the near positions than when they were present at the ends of the five-stimulus string $[F(1,11)=5.04, p<.05]$.

More important, however, was the significant two-way interactions between compatibility and distance [RT, 


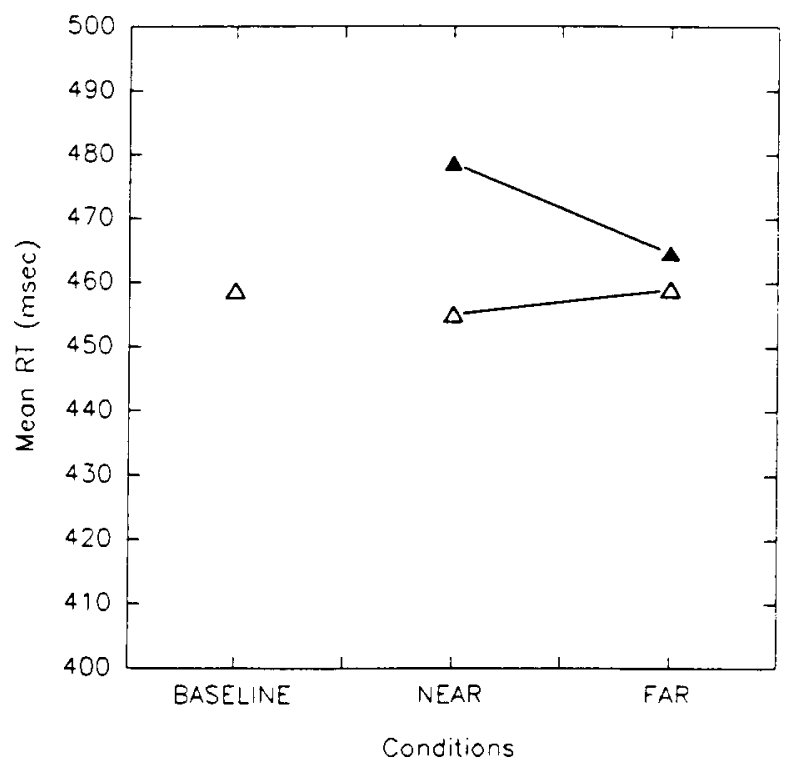

Figure 4. Average correct reaction times for each of the conditions in Experiment 2. Filled triangles indicate incompatible conditions; unfilled triangles indicate compatible conditions.

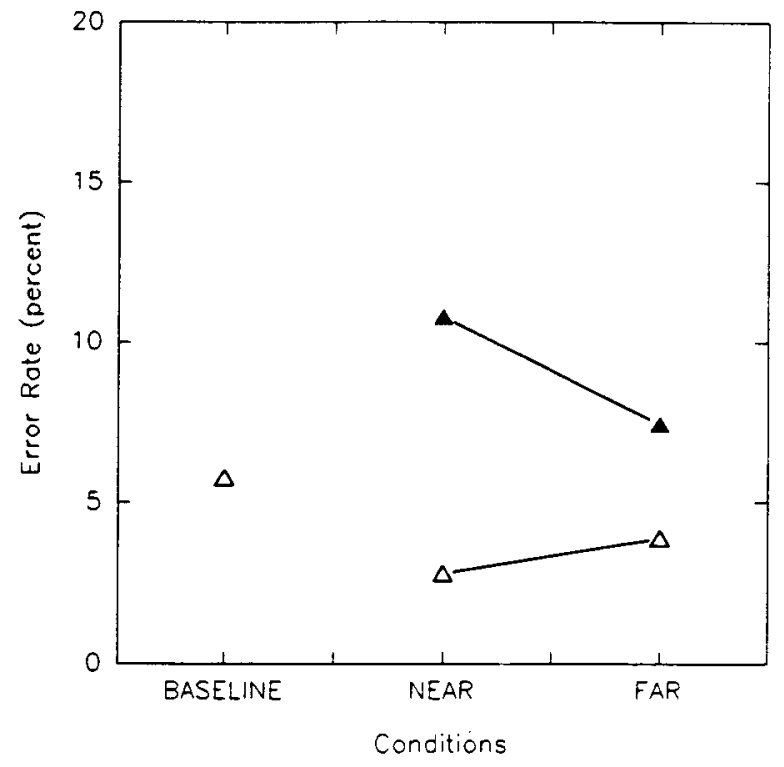

Figure 5. Average error rates for each of the conditions in Experiment 2. Filled triangles indicate incompatible conditions; unfilled triangles indicate compatible conditions.

$F(1,11)=17.89, p<.01 ;$ error rate, $F(1,11)=14.54$, $p<.01]$. Compatibility effects were larger for both RT and error rate when the compatible and incompatible distractors were located close to the target than when they were located at the far positions. This interpretation of the two-way interaction was supported by the finding of a significant difference between the near compatible and near incompatible conditions and the lack of a significant difference between the far compatible and far incompatible conditions for both RT and error rate. Thus, neither movement alone (Experiment 1) nor movement and color were able to override the proximity effect in the responsecompatibility paradigm.

Post hoc comparisons between the experimental conditions and the baseline revealed both costs and benefits in performance. RT was slower and error rates were higher in the near incompatible conditions than in the baseline condition. Conversely, RTs were faster and error rates were lower in the near compatible condition than in the baseline condition. RTs and error rates in the far conditions did not differ from those obtained in the baseline condition. Thus, both facilitation and interference effects were found when the response-compatible and response-incompatible stimuli were located next to the target. On the other hand, it appears that the subjects were able to ignore the letters located at the far positions despite the fact that they appeared in the same color and moved in the same direction and with the same velocity as the target. The RT and error rates in the far conditions were not statistically different from those in the baseline condition.

\section{Discussion}

The results of Experiment 2 do not support the notion of mandatory processing of movement. The redundant coding of the target and the far distractors with common color and movement failed to produce larger responsecompatibility effects than those obtained with the more proximate distractors. Our results are, however, consistent with Tsal and Lavie's (1988) suggestion that attention to spatial location is a general and mandatory process. Although the subjects were able to ignore the far distractors, they were not capable of excluding the near distractors during the processing of the centrally located target. These findings are also compatible with previous empirical research that has demonstrated the existence of a minimum focus of attention in which all stimuli are processed (B. A. Eriksen \& C. W. Eriksen, 1974; C. W. Eriksen \& Hoffman, 1972, 1973; C. W. Eriksen \& St. James, 1986). It appears that, in Experiment 2, the near distractors resided within this minimum focus and the far distractors were located beyond its boundaries.

\section{GENERAL DISCUSSION}

The original motivation for our research was to examine the nature of the grouping-by-movement phenomenon that was reported by Driver and Baylis (1989). We believed that their finding of a larger response-compatibility effect for distant distractors that moved in common with a target, relative to that found for stationary but more proximate distractors, was particularly important since it suggested the mandatory processing of stimuli with the same motion, even in a situation in which such processing would be detrimental to performance. Of course, another important aspect of their findings concerned the manner in which attention is deployed in the visual field. The demonstration that attention can be focused in several 
noncontiguous areas of the visual field is inconsistent with the class of space-based models of attention that postulate a single focus of attention (Broadbent, 1982; Downing \& Pinker, 1985; C. W. Eriksen \& St. James, 1986; Jonides, 1983; Posner, 1980; Remington \& Pierce, 1984). On the other hand, the demonstration of grouping by common movement is quite compatible with object- or grouping-based models of attention (Duncan, 1984; Kahneman \& Henik, 1984; Kramer \& Jacobson, 1991; Neisser, 1967), which postulate that attention is allocated to perceptual groups rather than areas of visual space.

Unfortunately, the results of our experiments were quite disappointing with regard to our failure to replicate Driver and Baylis's (1989) grouping-by-movement effect. In both of the experiments, the response-compatibility effects were significantly larger at the near distractor location than at the far location, indicating that common movement was having little effect on target processing. This pattern of results was obtained even when we redundantly coded the target and the far distractors with movement and color.

After conducting the first two experiments presented in this paper, we discovered a plausible explanation for the discrepant results: On page $\mathbf{8 4 0}$ of the Joumal of Experimental Psychology: Human Perception \& Performance (1989, Vol. 15), Driver and Baylis published an erratum to correct an error in their original manuscript. They indicated in the erratum that the movements actually took twice as long as had been originally stated. Thus, in three out of four experiments, the movements lasted $200 \mathrm{msec}$ rather than $100 \mathrm{msec}$.

Given that pursuit eye movements can begin with a latency of approximately $130 \mathrm{msec}$ (Carl \& Gellman, 1985; Gellman \& Carl, 1985; Robinson, Gordon, \& Gordon, 1986), it is possible that some portion of the groupingby-movement effects reported by Driver and Baylis (1989) may have been due to a tracking of the target and the far flankers by eye movements, rather than to attentional shifts. If this eye tracking occurred, it would have had the effect of stabilizing the target and the far distractors on the retina while smearing the image of the static near distractors. Thus, the grouping by common movement could be an epiphenomenon of the differential sensitivity of the visual system to the stabilized and smeared images.

In an effort to further investigate the eye-movement account of the grouping effect, we replicated our Experiment 1 with 12 different subjects and a movement time of $200 \mathrm{msec}$. If eye movements were responsible for some portion of the grouping effect found by Driver and Baylis (1989), we would expect to see a decrease or reversal of the near grouping effect that we reported in Experiment 1. That is, rather than finding a larger response-compatibility effect for the near distractors, we would expect to find a shift towards a larger response-compatibility effect for the far distractors. The results that we obtained, however, were identical to those found in the present Experiments 1 and 2 . The response-compatibility effect was significantly larger at the near-distractor locations than at the far-distractor locations.
Given our failure to find evidence in support of the eyemovement explanation for the grouping-by-movement effect, we decided to pursue one additional hypothesis. This hypothesis concerns the fact that while movement might have engendered the grouping of the target with the far distractors, the increased retinal eccentricity of the far relative to the near distractors might have had the opposite effect. Although common movement might be expected to increase the magnitude of the responsecompatibility effect for the far distractors, relative to the near distractors, the reduced acuity of the far distractors might be expected to reduce the impact of these stimuli on the processing of the target. Therefore, given the confound between target/distractor separation and retinal acuity, the grouping-by-movement effect demonstrated by Driver and Baylis (1989) might be a relatively fragile phenomenon.

In an effort to determine whether grouping by movement would override the effects of grouping by proximity in the absence of an acuity confound, we scaled the near and far distractors by the human cortical magnification factor suggested by Rovamo and Virsu (1979). This adjustment is based on changes in the scale of the mapping of visual field size to millimeters of visual cortex from the fovea to the periphery. Thus, the size of the distractors were increased, with a larger increase in size for the far distractors than for the near distractors, to compensate for decreases in acuity with increases in retinal eccentricity.

With the exception of the adjustment of the size of the near and far distractors, we replicated the DB conditions from Experiment 1 with 12 new subjects. The results were quite similar to those obtained in Experiment 1. The response-compatibility effect was significantly larger when the distractors were presented at the near locations than when they were presented at the far locations. Mean RTs for the near compatible, near incompatible, far compatible, and far incompatible conditions were $467,499,474$, $485 \mathrm{msec}$, respectively. The percent correct scores for the same conditions were 95.7, 88.4, 94.4, and 91.0.

In summary, despite our attempts to enhance the grouping of the centrally located target with the far distractors by redundantly coding these stimuli with common movement and color and by unconfounding target/distractor separation with acuity, we have been unable to replicate the grouping-by-movement effect reported by Driver and Baylis (1989). Although we do not know the source of the difference between our results and those reported by Driver and Baylis, it would appear that subjects are quite capable of selectively processing a target item that moves in the same direction and with the same velocity as a set of distractors.

\section{REFERENCES}

Banks, W. P., Bodinger, D., Illige, M. (1974). Visual detection accuracy and target-noise proximity. Bulletin of the Psychonomic Society, 2, 411-414. 
Banks, W. P., Punzmetal, W. (1976). Configural effects in visual information processing. Perception \& Psychophysics, 19, 361-367.

BArRer, P., Folkard, S. (1972). Reaction time under stimulus uncertainty with response certainty. Journal of Experimental Psychology, 93, 138-142.

Bashinski, H. S. , BaChaRACH, V. R. (1980). Enhancement of perceptual sensitivity as a result of selectively attending to spatial locations. Perception \& Psychophysics, 28, 241-248.

BECK, J., AMBLER, B. (1973). The effects of concentrated and distributed attention on peripheral acuity. Perception \& Psychophysics, 14, 225-230.

BrondBent, D. (1982). Task combination and selective intake of information. Acta Psychologica, 50, 253-290.

Bundersen, C., \& Pedersen, L. F. (1983). Color segregation and visual search. Perception \& Psychophysics, 33, 487-493.

Carl, J. R., \& Gellman, R. S. (1985). Human smooth pursuit: The response to conflicting velocity and position stimuli. Society for Neuroscience Abstracts, 11, 78.

Davis, E., Kramer, P., Graham, N. (1983). Uncertainty about spatial frequency, spatial position, or contrast of visual patterns. Perception \& Psychophysics, 33, 20-28.

Downing, C. J. (1988). Expectancy and visual-spatial attention: Effects on perceptual quality. Journal of Experimental Psychology: Human Perception \& Performance, 14, 188-202.

Downing, C. J., \& Pinker, S. (1985). The spatial structure of visual attention. In M. I. Posner \& O. Marin (Eds.), Attention and performance $X I$ (pp. 171-187). Hillsdale, NJ: Erlbaum.

Driver, J., \& BAYLIS, G. C. (1989). Movement and visual attention: The spotlight metaphor breaks down. Joumal of Experimental Psychology: Human Perception \& Performance, 15, 448-456.

DUNCAN, J. (1984). Selective attention and the organization of visual information. Journal of Experimental Psychology: General, 113, 501-517.

Duncan, J., Humphreys, G. W. (1989). Visual search and stimulus similarity. Psychological Review, 96, 433-458.

Eruxsen, B. A., ERuksen, C. W. (1974). Effects of noise letters upon the identification of a target letter in a nonsearch task. Perception \& Psychophysics, 16, 143-149.

Eriksen, C. W., Hoffman, J. E. (1972). Temporal and spatial characteristics of selective encoding from visual displays. Perception \& Psychophysics, 12, 201-204.

Eriksen, C. W., Hoffman, J. E. (1973). The extent of processing noise elements during selective encoding from visual displays. Perception \& Psychophysics, 14, 155-160.

ERIKSEN, C. W., ST. JAMES, J. D. (1986). Visual attention within and around the field of focal attention: A zoom lens model. Perception \& Psychophysics, 40, 225-240.

ERIKSEN, C. W., Y YeH, Y. Y. (1985). Allocation of attention in the visual field. Joumal of Experimental Psychology: Human Perception \& Performance, 11, 583-597.

Fryklund, I. (1975). Effects of cued-set spatial arrangement and target background similarity in the partial report paradigm. Perception \& Psychophysics, 17, 375-386.

GARNER, W. R. (1987). Location and color as cueing dimensions in contingent classification. Perception \& Psychophysics, 41, 202-210.

GAtTI, S. V., E EGETH, H. E. (1978). Failure of spatial selectivity in vision. Bulletin of the Psychonomic Society, 11, 181-184.

Gellman, R. S., CARL, J. R. (1985). Human smooth pursuit: Early response to sudden changes in target velocity. Society for Neurosciences Abstracts, 11, 79.

HagenaAR, R., \& VAN der Heidden, A. H. C. (1986). Target-noise separation in visual selective attention. Acta Psychologica, 62, 161-176.

Harms, L., \& Bundersen, C. (1983). Color segregation and selective attention in a nonsearch task. Perception \& Psychophysics, 33, 11-19.

Hoffman, J., Nelson, B., Houck, M. (1983). The role of attentional resources in automatic detection. Cognitive Psychology, 15, $379-410$.
Humphreys, G. W. (1981). Flexibility of attention between stimulus dimensions. Perception \& Psychophysics, 30, 291-302.

Ishari Color Blindness Test (1989). Tokyo: Kanchara.

JONIDES, J. (1983). Further toward a model of the mind's eye movement. Bulletin of the Psychonomic Society, 21, 247-250.

KAHNEMAN, D., HENIK, A. (1977). Effects of visual grouping on immediate recall and selective attention. In S. Domic (Ed.), Attention and performance VII (pp. 307-332). Hillsdale, NJ: Erlbaum.

Kahneman, D., Henik, A. (1981). Perceptual organization and attention. In M. Kubovy \& J. R. Pomerantz (Eds.), Perceptual organization (pp. 181-211). Hillsdale, NJ: Erlbaum

Kahneman, D. , Treisman, A. (1984). Changing views of attention and automaticity. In R. Parasuraman \& R. Davies (Eds.), Varieties of attention (pp. 29-62). New York: Academic Press.

Kramer, A. F., Jacobson, A. (1991). Perceptual organization and focused attention: The role of objects and proximity in visual processing. Perception \& Psychophysics.

Kramer, A. F., Wickens, C. D., Donchin, E. (1985). Processing of stimulus properties: Evidence for dual-task integrality. Joumal of Experimental Psychology: Human Perception \& Performance, 11, 393-408.

LABERGE, D., Brownstern, L. S. (1974). Control of visual processing by color coding. Bulletin of the Psychonomic Society, 2, 417-418.

LAPPIN, J. (1967). Attention in the identification of stimuli in complex visual displays. Journal of Experimental Psychology, 75, 321-328.

Mangun, G. R. R., Hillyard, S. A. (1987). The spatial allocation of visual attention as indexed by event-related brain potentials. $\mathrm{Hu}$ man Factors, 29, 195-211.

Neisser, U. (1967). Cognitive psychology. Englewood Cliffs, NJ: Prentice Hall.

NisSEN, M. J. (1985). Accessing features and objects: Is location special? In M. I. Posner \& O. S. Marin (Eds.), Attention and performance $X I$ (pp. 205-219). Hillsdale, NJ: Erlbaum.

Pomerantz, J. R., Prustach, E. A. (1989). Emergent features, attention, and perceptual glue in visual form perception. Joumal of Experimental Psychology: Human Perception \& Performance, 4, 635-649.

Pomerantz, J. R., \& Schwartzberg, S. D. (1975). Grouping by proximity: Selective attention measures. Perception \& Psychophysics, 18, 355-361.

PosNer, M. I. (1980). Orienting of attention. Quanerly Journal of Experimental Psychology, 32, 3-25.

Posner, M. I., Snyder, C. R. R., \& DAvidson, B. J. (1980). Attention and the detection of signals. Journal of Experimental Psychology: General, 109, 160-174.

Prinzmetal, W. (1981). Principles of feature integration in visual perception. Perception \& Psychophysics, 30, 330-340.

Remington, R. W., * Pierce, L. (1984). Moving attention: Evidence for time invariant shifts of visual selective attention. Perception \& Psychophysics, 35, 393-399.

Robinson, D. A., Gordon, J. L., \& Gordon, S. E. (1986). A model of the smooth pursuit eye movement system. Biological Cybernetics, 55, 43-57.

Rovamo, J., VIRSU, V. (1979). An estimation and application of the human cortical magnification factor. Experimental Brain Research, 37, 495-510.

Shulman, G. L., Remington, R. W., a Mclean, J. P. (1979). Moving attention through visual space. Journal of Experimental Psychology: Human Perception \& Performance, 5, 522-526.

Treisman, A. M., Gelade, G. (1980). A feature-integration theory of attention. Cognitive Psychology, 12, 97-136.

Treisman, A. M., Gormican, S. (1988). Feature analysis in early vision: Evidence from search asymmetries. Psychological Review, 95, 15-48.

Treisman, A., Kahneman, D., Burkell, J. (1983). Perceptual objects and the cost of filtering. Perception \& Psychophysics, 33, 527-532. 
TSAL, Y., LAVIE, N. (1988). Attending to color and shape: The special role of location in selective visual processing. Perception \& Psychophysics, 44, 15-21.

VoN WRIGHT, J. M. (1968). Selection in visual immediate memory. Quanerly Journal of Experimental Psychology, 20, 62-68.

YNTEMA, D. B. (1963). Keeping track of several things at once. $\mathrm{Hu}$ man Factors, 5, 7-17.

\section{NOTES}

1. All post hoc comparisons reported in this article were computed with a Bonferoni $t$ test and were significant at $p<.05$.

2. We have suggested that the larger response-compatibility effect at the near distractor location of the proximate condition, relative to that of the DB condition, can be attributed to the enhanced grouping of the distractors and the target on the proximate trials. This interpretation is based on the notion that items that are located in close proximity will be more strongly grouped than items that are more widely spaced (Pomerantz \& Schwaitzberg, 1975). However, given that the more widely spaced distractors are also located at more peripheral retinal locations, it is conceivable that the smaller response-compatibility effect in the DB condition might be due to differences in acuity rather than target/distractor grouping. To evaluate this alternative interpretation, we conducted an experiment in which 12 subjects were instructed to make one response if they saw an $X$ or a $Y$ on the display and another response if they saw an $S$ or a $C$. On each trial, the subjects were presented with a fixation cross followed by either an $X$, a Y, an S, or a C. The stimuli appeared randomly at either $0.4^{\circ}$ or $0.6^{\circ}$ from fixation (e.g., at the proximate and DB distractor locations, respectively) on the right or left of the display. Each subject performed six blocks of 100 trials. The mean RTs and accuracies differed by $2 \mathrm{msec}$ and $0.7 \%$ for the $0.4^{\circ}$ and $.6^{\circ}$ locations (proximate condition, RT $=458 \mathrm{msec}$, accuracy $=95.0 \%$; DB condition, $R T=460 \mathrm{msec}$, accuracy $=95.7 \%$ ). These small and nonsignificant differences between the $0.4^{\circ}$ and $0.6^{\circ}$ locations suggest that reduced compatibility effects in the DB condition cannox be atributed to acuity differences between the DB and proximate trials.

3. To determine if there were differential color effects we initially performed split-plot ANOVAs with color as the between-subjects factor and compatibility and distance as within-subjects factors. Neither the main effect of color nor its interaction with other variables attained statistical significance. Therefore, we collapsed across color in the subsequent RT and error rate ANOVAs.

(Manuscript received April 15, 1991; revision accepted for publication July 23,1991 .)

\section{Notice}

\section{Call for Assistance in The Compilation of a History of the Psychonomic Society}

The Governing Board of the Psychonomic Society is pleased to announce that Robert C. Bolles has agreed to serve as the first Historian of the Society.

All members who might have information relevant to this undertaking are invited to send it directly to Dr. Bolles. Founding members and those who attended the early meetings are especially encouraged to record their reminiscences. While Dr. Bolles hopes to collect as much information as possible relevant to the history of the Society, he will concentrate first on the early history.

Dr. Bolles's address is Department of Psychology, University of Washington, Seattle, Washington 98195 (phone: 206-543-2631). 